\title{
Bruton's tyrosine kinase regulates macrophage-induced inflammation in the diabetic kidney via NLRP3 inflammasome activation
}

\author{
JING ZHAO $^{1}$, JUAN CHEN ${ }^{1}$, YUAN-YUAN LI $^{1}$, LING-LING XIA $^{2 *}$ and YONG-GUI WU ${ }^{1 *}$ \\ Departments of ${ }^{1}$ Nephropathy and ${ }^{2}$ Infectious Diseases, The First Affiliated Hospital of \\ Anhui Medical University, Hefei, Anhui 230022, P.R. China
}

Received January 22, 2021; Accepted June 18, 2021

DOI: $10.3892 /$ ijmm.2021.5010

\begin{abstract}
It has been previously reported that macrophages may be involved in diabetic nephropathy (DN) development. Furthermore, Bruton's tyrosine kinase (BTK) may participate in macrophage activation and lead to the release of inflammatory mediators. The main aim of the present study was to analyze the association between renal BTK expression and clinical indicators. Moreover, BTK knockout mice were used to establish a diabetic model for further research. The results demonstrated that BTK was activated in the kidneys of patients with DN and was associated with the progression of proteinuria, creatinine levels, estimated glomerular filtration rate and pathological changes in the kidneys of patients with DN. Furthermore, BTK knockout was observed to reduce urinary protein excretion, alleviate renal injury and decrease renal inflammation in diabetic mice. This protection may be attributed to BTK-induced suppression of the activation of the Nod-like receptor (NLR) family pyrin domain containing 3 inflammasome. Collectively, it has been demonstrated in the present study that BTK may be a potential target for DN treatment.
\end{abstract}

\section{Introduction}

Diabetic nephropathy (DN) is a severe microvascular complication in diabetic patients and has been the main cause of

Correspondence to: Professor Ling-Ling Xia, Department of Infectious Diseases, The First Affiliated Hospital of Anhui Medical University, 218 Jixi Road, Hefei, Anhui 230022, P.R. China E-mail: 13966684365@163.com

Professor Yong-Gui Wu, Department of Nephropathy, The First Affiliated Hospital of Anhui Medical University, 218 Jixi Road, Hefei, Anhui 230022, P.R. China

E-mail: wuyonggui@medmail.com.cn

Key words: diabetic nephropathy, Bruton's tyrosine kinase, Nod-like receptor family pyrin domain containing 3 , macrophage, inflammation end-stage renal disease (1). DN is mainly manifested as the thickening of the glomerular basement membrane (GBM), the proliferation of mesangial cells and extracellular matrix, and renal tubulointerstitial fibrosis (2). Furthermore, its pathogenesis is complex, involving hereditary, metabolic and hemodynamic changes, as well as inflammation. A recent study has reported the correlation between DN and inflammation (3). Macrophages are a type of immune cell and are derived from monocytes. Macrophages have multiple functions, such as phagocytosing cell fragments, releasing chemokines factors and secreting inflammatory mediators (4). Furthermore, it has been established that macrophages play a key role in the occurrence and development of DN $(5,6)$.

Bruton's tyrosine kinase (BTK) is an intracellular non-receptor tyrosine kinase, and is one of the five members of the Tec tyrosine kinase family (7). BTK is expressed in myeloid cells, such as macrophages and tissue plasma cells. Moreover, BTK signaling molecules have been well-documented and it has been considered as an important signal in immunoregulation (8). It has recently demonstrated that BTK is crucial for the proliferation, development and differentiation of B cells (9). In addition, BTK may be involved in human innate immunity, particularly in relation to B cell and macrophage involvement (10). It has also been demonstrated that BTK can conduct cell signals by regulating Toll-like receptor (TLR)2 and TLR4 in macrophages (11). Previous studies have proved that BTK-regulated inflammation is involved in various renal diseases, such as IgA nephropathy and Lupus nephritis $(12,13)$. The BTK inhibitor PCI-32765, also known as ibrutinib, has been widely used as an antitumor drug for the treatment of chronic lymphocytic leukemia (14). It has been reported that ibrutinib can reduce MAPK and NF- $\kappa$ B pathway activation, and then decrease the release of inflammatory mediators in macrophages (15). Moreover, a recent study demonstrated that a BTK inhibitor may serve as an effective therapeutic strategy in severe COVID-19, due to its excellent anti-inflammatory effect (16).

The inflammasome is a multi-protein complex expressed in myeloid cells, and is mainly composed of sensors, apoptosis-associated speck like protein containing a caspase recruitment domain (ASC), and the caspase protease. Inflammasomes exist in the cytoplasm of multiple cell types 
and have the ability to induce the innate immune responses by sensing damage signals and microbial attacks (17). The Nod-like receptor (NLR) family pyrin domain containing 3 (NLRP3) inflammasome is the most widely studied inflammasome, and is composed of NLRP3, ASC and pro-caspase-1 (18). Moreover, the NLRP3 inflammasome can be activated and is known to participate in the development of inflammation by cleaving the inactive cytokines, IL-1 $\beta$ precursor and IL-18 precursor, into active IL-1 $\beta$ and IL-18 $(19,20)$. Recent studies have reported that the activation of the NLRP3 inflammasomes plays an important role in diabetic kidney disease (DKD) $(21,22)$. Additionally, inflammasome activation marker expression levels, such as caspase-1, IL-1 $\beta$ and IL-18, have been shown to be positively associated with the severity of albuminuria in patients with DN $(23,24)$.

Although the therapeutic potential of NLRP3 is undisputed, currently no clinically approved therapies exist that target the NLRP3 inflammasome directly, at least to the best of our knowledge (25). Recently, BTK has attracted increased attention as a regulator of NLRP3. It has been demonstrated that the inhibition of BTK can reduce the inflammatory response by decreasing the activation of the NLRP3 inflammasome in numerous diseases, such as ischemic brain injury, diet-induced metabolic inflammation and polymicrobial sepsis (26-28). However, the role of BTK in DN remains to be elucidated. On this basis, the present study aimed to investigate the effect of BTK on DN and its association with the NLRP3 inflammasome in the kidneys.

\section{Materials and methods}

Recruitment. A total of 49 patients with type $2 \mathrm{DN}$ and 18 healthy individuals were recruited from the Department of Nephrology in The First Affiliated Hospital of Anhui Medical University between January 1, 2017 and January 1, 2020. All patients exhibited no fever symptoms or infectious diseases, and were diagnosed via renal biopsy. Renal tissue sections from patients with DN were provided by the Kidney Pathology Center at The First Affiliated Hospital of Anhui Medical University. The para-carcinoma tissues from patients with renal carcinoma was used as the control group (29). The clinical characteristics of all the study subjects are presented in Tables SI and SII. Serum samples from patients and healthy individuals were provided by the Clinical Laboratory of The First Affiliated Hospital of Anhui Medical University. All the patients and healthy individuals had signed informed consent forms and all experiments were approved by the Ethics Committee of Anhui Medical University (approval number: 5101309).

Animals and experimental groups. A total of 28 male C57BL/6J mice (age, 6-8 weeks; weight, 18-22 g) were purchased from the Experimental Animal Center of Nanjing Medical University. The animal experiment was performed in accordance with guidelines, 'Principles of Laboratory Animal Care and Use in Research' (Ministry of Health, Beijing, China). The experiments were approved by the Anhui Medical University Ethics Committee (approval number: 2020064). A total of 30 BTK-floxed (Macrophage-specific knockout) mouse frozen embryos were purchased from The European Mouse Mutant Archive and resuscitated at the Experimental Animal
Center of Nanjing Medical University. The embryos were removed from liquid nitrogen and were resuscitated in a water bath for $2 \mathrm{~min}$ at $35^{\circ} \mathrm{C}$, then immediately placed in a Petri dish with thawing solution (0.1 M sucrose PBS with $6 \%$ glycerin) for $1 \mathrm{~min}$, followed by diluent solution (0.1 M sucrose PBS with $3 \%$ glycerin) for $3 \mathrm{~min}$. The embryos were then transferred to the washing solution ( $0.1 \mathrm{M}$ sucrose PBS with $0 \%$ glycerin) for 3 min twice. The embryos were then cultured in HTF medium (cat. no. MR-070-D; EMD Millipore) in an incubator at $37^{\circ} \mathrm{C}$ with $5 \% \mathrm{CO}_{2}$. Following $24 \mathrm{~h}$ of culture at $37^{\circ} \mathrm{C}$, the normal embryos were selected and transplanted into the uterus of 15 female C57BL/6J mice (age, 6-8 weeks; weight, 18-22 g, obtained from the Experimental Animal Center of Nanjing Medical University) $(30,31)$. The offspring from the female mice (the BTK-floxed mice) were then crossed with 6 male and 9 female CMV-cre transgenic mice (age, 6-8 weeks; weight, 18-22 g; C57BL/6J genetic background, obtained from the Experimental Animal Center of Nanjing Medical University), and the offspring were self-crossed to obtain BTK knockout mice $\left(\mathrm{BTK}^{-/-}\right.$mice).

Streptozotocin (STZ) was purchased from Sigma-Aldrich (Merck KGaA). The mice were administered STZ daily, at a dose of $50 \mathrm{mg} / \mathrm{kg}$ of body weight for 5 days. In total, 28 mice were randomly divided into 4 groups as follows: i) Wild-type (WT) group (WT littermate C57BL/6J mice; $\mathrm{n}=6$ ); ii) STZ group (WT littermate C57BL/6J mice + STZ; $\mathrm{n}=8)$; iii) $\mathrm{BTK}^{-/-}$group $(\mathrm{n}=6)$; and iv) $\mathrm{BTK}^{-/}+\mathrm{STZ}$ group $\left(\mathrm{BTK}^{--}+\mathrm{STZ} ; \mathrm{n}=8\right)$. All mice were maintained under standard feeding conditions (temperature, $20 \pm 2^{\circ} \mathrm{C}$; humidity, $45-55 \%$; 12-h light/dark cycle) and were granted free access to food and water. After 12 weeks of rearing, all mice were anesthetized with an intraperitoneal injection of sodium pentobarbital (50 mg/kg body weight) and euthanized via exsanguination by drawing $1.2 \mathrm{ml}$ blood. All mice were observed for $20 \mathrm{~min}$ to verify death, according to the criterion of breathing cessation and cardiac arrest. The experimental humane endpoints that were established in the present study, included loss of appetite, rapid weight loss ( $>20 \%$ of body weight within a week), severe infections, weakness and organ failure.

Genotype identification. After euthanizing the mice, $3 \mathrm{~mm}$ mouse tail was obtained and used for DNA extraction (cat. no. 10185ES50, Shanghai Yeasen Biotechnology Co., Ltd.). Following amplification in a QuantStudio ${ }^{\mathrm{TM}} 6$ Flex Real-Time PCR System (Thermo Fisher Scientific, Inc.), gene expression was detected by $1.5 \%$ agarose gel electrophoresis. The PCR conditions were as follows: $95^{\circ} \mathrm{C}$ for $5 \mathrm{~min}$, followed by 40 cycles of $95^{\circ} \mathrm{C}$ for $30 \mathrm{sec}, 58^{\circ} \mathrm{C}$ for $30 \mathrm{sec}$ and $72^{\circ} \mathrm{C}$ for $30 \mathrm{sec}$. The primers (Shanghai Sangon Biotech Co., Ltd.) were as follows: BTK-FRT-tF1, CTGCATAAGGCAGGTGCCACT AA, and BTK-FRT-tR1, CATCAGAAGCAGGCCACCCA; BTK-loxP-tF1, TTGCATAAAGGCAGCA ATACAACAG, and BTK-loxP-tR1, TAGCTCCAGAACTCAATGACAAAG A; lacZ3F4, CCGGTCGCTACCATTACCAGT; IMP C-loxptR, ATGGCGAGCTCAGACCATAAC; Cre KT 119, TGCCACGACCAAGTGACAGCAATG, and Cre KT 120, ACCAGAGACGGAAATCCATCGCTC.

Immunohistochemical staining. The paraffin-embedded kidney tissues from mice were cut into $3-\mu \mathrm{m}$-thick sections. 
All sections were deparaffinized, rehydrated and subjected to antigen retrieval in $0.01 \mathrm{M}$ citrate buffer $(\mathrm{pH} 6.0)$ by microwaving. After blocking with $10 \%$ goat serum (cat. no. SL038; Beijing Solarbio Science \& Technology Co., Ltd.) at room temperature, the sections were incubated with anti-CD68 (cat. no. ab213363; 1:300; Abcam), anti-BTK (1:200; cat. no. 8547; Cell Signaling Technology, Inc.), anti-F4/80 (1:200; cat. no. ab111101; Abcam), anti-collagen (Col)-IV (1:200; cat. no. ab236640; Abcam), anti-fibronectin (FN; 1:200; cat. no. ab2413; Abcam), anti-Wilms Tumor 1 (WT1) transcription factor (WT1; 1:200; cat. no. ab267377; Abcam) and anti-Nephrin (1:200; cat. no. ab216341; Abcam) antibodies overnight at $4^{\circ} \mathrm{C}$. Secondary biotin-labeled goat anti-mouse/rabbit IgG antibodies (1:200; cat. no. PV-6000; OriGene Technologies, Inc.) were then added for $2 \mathrm{~h}$ at room temperature. Finally, 3,3-diaminobenzidine (cat. no. ZLI-9017; Sigma-Aldrich; Merck KGaA) and hematoxylin at room temperature were used for staining. All images were captured with a Zeiss microscope (Zeiss AG) and analyzed using ImageJ 1.0 software (National Institutes of Health). Six fields were selected for statistical analysis in each sample. The mean value was calculated and used as the final sample of the immunohistochemistry data. The mean values of all samples were used for final statistical analysis.

ELISA. The levels of TNF- $\alpha$, IL-1 $\beta$ and monocyte chemoattractant protein-1 (MCP-1) in serum were detected with the use of human and mouse ELISA kits (cat. no. P06804, P10749, P10148; RayBiotech, Inc.). The urinary albumin excretion rate (UAER) was detected using a mouse albumin ELISA kit (cat. no. ab108792, Abcam). The detection methods were executed according to the manufacturer's instructions.

Detection of HbAlc and blood glucose. HbAlc was detected using the Glycosylated hemoglobin A1c Assay kit (cat. no. H464-1, Nanjing Jiancheng Bioengineering Institute). The blood glucose levels were detected using the glucose meter (Contour TS, Bayer). The detection methods were executed according to the manufacturer's instructions.

Western blot (WB) analysis. Renal tissues were lysed in RIPA lysis buffer (cat. no. P0013B, Beyotime Institute of Biotechnology). The mixture was centrifuged at 15,294 x g at $4^{\circ} \mathrm{C}$ for $30 \mathrm{~min}$ and then the pellet was removed. Protein samples were separated via 10 or $15 \%$ SDS-PAGE, and transferred to the nitrocellulose membrane (cat. no. HATF00010, Merck KGaA). The band distribution was observed via Ponceau staining. The membrane was then blocked with 5\% skim milk powder (containing TBS-Tween-20) at room temperature for $2 \mathrm{~h}$, followed by incubation with the following primary antibodies at $4^{\circ} \mathrm{C}$ for $12 \mathrm{~h}$ : Anti- $\beta$-actin $(1: 35,000$, cat. no. 66009-1-Ig; ProteinTech Group, Inc.), anti-TNF- $\alpha$ (1:1,000; cat. no. ARG10158; arigo Biolaboratories Corp.), anti-IL-1 $\beta$ (1:1,000; cat. no. 12242; Cell Signaling Technology, Inc.), anti-MCP-1 (1:1,000; cat. no. ARG57649; arigo Biolaboratories Corp.), anti-BTK (1:1,000; cat. no. ab25971; Abcam), anti-phosphorylated (p)-BTK (1:1,000; cat. no. 87457; Cell Signaling Technology, Inc.), anti-inducible nitric oxide synthase (iNOS; 1:1,000; cat. no. ab178945; Abcam),

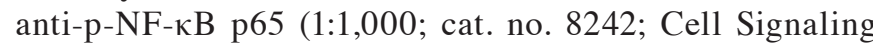

Technology, Inc.), anti-NF-кB p65 (1:1,000; cat. no. 8242; Cell Signaling Technology, Inc.), anti-nicotinamide adenine dinucleotide phosphate oxidase 1 (NOX1) (cat. no. ab131088; 1:1,000; Abcam), anti-nicotinamide adenine dinucleotide phosphate oxidase 4 (NOX4) (1:1,000; cat. no. bs-1091R; Beijing Boaosen Biotechnology, Co., Ltd.), anti-NLRP3 (1:1,000; cat. no. ab214185; Abcam), anti-caspase-1 (1:1,000; cat. no. ab138483; Abcam) and anti-ASC (1:200; cat. no. sc-376916; Santa Cruz Biotechnology, Inc.). After washing, the membrane was incubated with HRP-labeled secondary antibodies (cat. nos. SA00001-1 and SA00001-2; 1:2,000; ProteinTech Group, Inc.) at room temperature for $1 \mathrm{~h}$. The signals were detected with the use of a chemiluminescent gel imaging system and the relative ratio was semi-quantified using ImageJ 1.0 software (National Institutes of Health).

Reverse transcription-quantitative (RT-qPCR). TRIzol ${ }^{\circledR}$ reagent (Thermo Fisher Scientific, Inc.) was used to extract the total RNA in renal tissues. cDNA was synthesized from total RNA using a Reverse Transcription kit (Vazyme Biotech Co., Ltd.). cDNA was amplified and detected in a QuantStudio ${ }^{\mathrm{TM}} 6$ Flex Real-Time PCR System (Thermo Fisher Scientific, Inc.) using the miScript SYBR-Green PCR kit (Qiagen $\mathrm{GmbH}$ ) to determine the quantity of mRNA. The conditions were as follows: $95^{\circ} \mathrm{C}$ for $10 \mathrm{~min}$, followed by 40 cycles of $95^{\circ} \mathrm{C}$ for $10 \mathrm{sec}$, and $60^{\circ} \mathrm{C}$ for $20 \mathrm{sec}$. The expression levels of all genes were standardized with the reference gene GAPDH using the $2^{-\Delta \Delta C q}$ method (32). The primer sequences used were selected according to the previous studies $(15,33)$.

Histological staining. The kidney tissue was fixed in $4 \%$ paraformaldehyde for $16 \mathrm{~h}$ at room temperature and then dehydrated through an ethanol gradient. Subsequently, the kidney tissue was paraffin-embedded and cut into $3-\mu \mathrm{m}$-thick sections. The sections were dewaxed and stained with periodic acid-Schiff (PAS) staining kit (cat. no. G1281; Beijing Solarbio Science \& Technology Co., Ltd.) and the Masson's staining kit (cat. no. G1340; Beijing Solarbio Science \& Technology Co., Ltd.). The staining methods were executed according to the manufacturer's instructions. All images were acquired using a light microscope (BX51, Olympus Corporation). The glomerular mesangial expansion, tubulointerstitial injury index and fibrosis area were evaluated and graded using Image J 1.0 software (National Institutes of Health).

Transmission electron microscopy (TEM). Kidney tissue was quickly placed in $2 \%$ glutaraldehyde for $24 \mathrm{~h}$. The tissue was then fixed with $1 \%$ acetic acid at room temperature for $2 \mathrm{~h}$, followed by acetone $(50,70,80,90$ and $100 \%$; twice for $15 \mathrm{~min}$ each time) for dehydration; sections were embedded in epoxy resin. The resin block was cut into ultra-thin sections of $70 \mathrm{~nm}$, stained with lead citrate at $37^{\circ} \mathrm{C}$ and then observed under a transmission electron microscope (H-7700; Hitachi, Ltd.). GBM thickness and foot process fusion were analyzed using ImageJ 1.0 software (National Institutes of Health).

Statistical analysis. Data were statistically analyzed using SPSS 23.0 (IBM Corp.). Normally distributed data are presented as the mean $\pm \mathrm{SD}$, and non-normally distributed data were expressed as the median (p25, p75). Pearson's test 
Table I. Clinical indicators of mice $(\mathrm{n}=28)$.

\begin{tabular}{lcccc}
\hline Clinical indicator & WT & STZ & BTK $^{-/-}$ & BTK $^{-/}+$STZ $^{-1}$ \\
\hline Body weight & $28.88 \pm 0.57$ & $22.56 \pm 1.93^{\mathrm{a}}$ & $27.16 \pm 1.02$ & $23.66 \pm 1.35$ \\
Kidney/body ratio $(\%)$ & $0.62 \pm 0.04$ & $1.00 \pm 0.11^{\mathrm{a}}$ & $0.70 \pm 0.06$ & $0.97 \pm 0.06$ \\
BG $($ mmol/l) & $6.51 \pm 0.68$ & $22.17 \pm 1.69^{\mathrm{a}}$ & $7.22 \pm 0.60$ & $22.76 \pm 2.85$ \\
HbA1c $(\%)$ & $4.42 \pm 0.65$ & $6.98 \pm 0.72^{\mathrm{a}}$ & $4.77 \pm 0.89$ & $7.14 \pm 1.10$ \\
UAER $(\mu \mathrm{g} / 24 \mathrm{~h})$ & $28.41 \pm 14.48$ & $449.87 \pm 101.43^{\mathrm{a}}$ & $26.31 \pm 18.22$ & $304.39 \pm 173.74^{\mathrm{b}}$ \\
\hline
\end{tabular}

All the data are expressed as the mean \pm SD. Significant differences were analyzed by one-way ANOVA with Bonferroni's post hoc analysis. ${ }^{\mathrm{a}} \mathrm{P}<0.05$ vs. WT group; ${ }^{\mathrm{P}} \mathrm{P}<0.05$ vs. STZ group. BG, blood glucose; HbA1c, haemoglobin A1C; UAER, urinary albumin excretion rate.

was used to analyze the correlation between normal distribution data. Spearman's test was used to analyze the correlation between non-normal distribution data. The homogeneity of variance was tested using the Levene method. Differences between two groups were assessed using an independent samples t-test. For multiple group comparisons, the significant differences were analyzed by one-way ANOVA, followed by Bonferroni's post hoc analysis. $\mathrm{P}<0.05$ was considered to indicate a statistically significant difference.

\section{Results}

BTK is markedly activated in renal macrophages in the kidneys of patients with DN. In the present study, the clinical indicators of 49 patients with type $2 \mathrm{DN}$ and 18 healthy individuals were collected from the Department of Nephropathy and Clinical Laboratory at the First Affiliated Hospital of Anhui Medical University. The results demonstrated that the DN group had significantly higher levels of haemoglobin A1c, systolic blood pressure (BP), diastolic BP, mean arterial pressure, proteinuria, serum creatinine, blood urea nitrogen, estimated glomerular filtration rate (eGFR) and serum uric acid (Table SI). The role of macrophage-mediated inflammation was evaluated by ELISA and immunohistochemistry in patients with DN. The data revealed that the TNF- $\alpha$, IL- $1 \beta$ and MCP-1 serum levels were significantly increased in the DN group (Fig. S1A). Additionally, the TNF- $\alpha$ and CD68 expression levels were significantly increased in the renal tissue from the DN group (Fig. S1B and C). This indicated that patients with DN exhibit more severe inflammation and macrophage infiltration in the kidneys. Moreover, the results also revealed that the DN group exhibited an increased BTK activation in comparison with the control group (Fig. 1A).

To further observe the activation of BTK in renal macrophages, CD68 was used to label macrophages followed by co-staining with BTK and p-BTK. The data indicated that the CD68 expression levels were increased in the DN group in comparison with the control group. Moreover, the BTK and p-BTK expression levels were increased in DN group (Fig. 1B). Furthermore, iNOS and NLRP3 were also notably activated in renal macrophages in the kidneys of patients with DN (Fig. S2). This suggested that macrophages exhibited an increased BTK activation in the kidneys of patients with DN; thus, the activation of the NLRP3 inflammasome may be involved in this process.
BTK expression is correlated with clinical indicators and pathological changes in the kidneys of patients with DN. Correlation analysis was conducted to determine the correlation between BTK expression and clinical indicators in patients with DN. The data demonstrated that the expression level of BTK positively correlated with proteinuria and serum creatinine levels, and negatively correlated with eGFR levels (Fig. 2A-F). The pathological scores of all renal patient tissues were also assessed (Table SII). The results depicted that the expression level of BTK in the glomerulus correlated with the Tervaert classification. In addition, renal BTK expression in the tubulointerstitium also correlated with interstitial fibrosis, the tubular atrophy score and the interstitial inflammation score (Fig. 2G-I). This indicated that BTK expression was significantly associated with disease progression in patients with DN.

BTK knockout alleviates renal injury in mice with STZ-induced diabetes. To further investigate the effects of BTK on DN, BTK knockout mice were used to establish a diabetic model. Mouse genotyping was initially performed (Fig. S3). After 12 weeks of rearing, the general indicators and metabolic parameters were measured in all mice. HbA1c was detected using an assay kit. The blood glucose levels were detected using a Bayer glucose meter. UAER was detected using a mouse albumin ELISA kit. The results identified that in comparison with the WT group, blood glucose, HbAlc, kidney/body weight and urinary protein excretion in the STZ group were significantly increased, while the mouse body weight was significantly decreased. The level of blood glucose, HbA1c, body weight and the kidney/body ratio exhibited no changes in the $\mathrm{BTK}^{-/}+\mathrm{STZ}$ group compared with the STZ group. However, the UAER was significantly reduced when BTK was knocked out (Table I). These findings suggest that BTK knockout may protect mice from diabetic kidney injury.

Subsequently, the changes in mouse renal injury were detected. PAS staining was used to observe the pathologic changes in mouse kidneys and Masson's trichrome staining was used to evaluate the fibrosis area. The results demonstrated that the STZ group exhibited more severe mesangial expansion and proliferation than the WT group; this was significantly reversed in the $\mathrm{BTK}^{-/}+\mathrm{STZ}$ group (Fig. 3A). However, no marked differences amongst groups was observed as regards the fibrosis area (Fig. S4). ColIV and FN were used to observe the proliferation of the mesangial matrix, while 
A

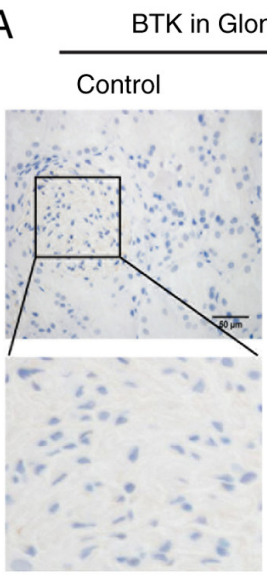

B

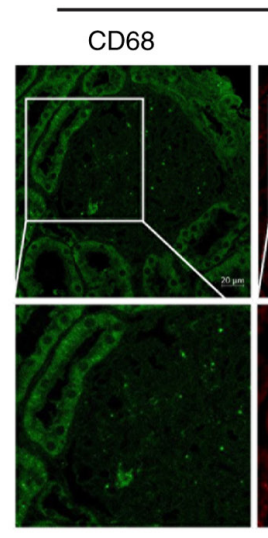

CD68
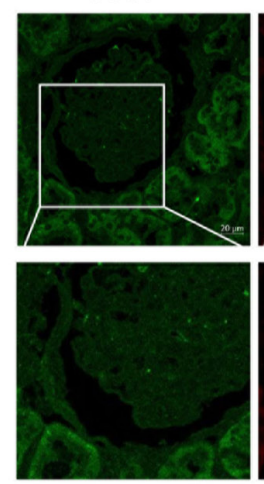

BTK
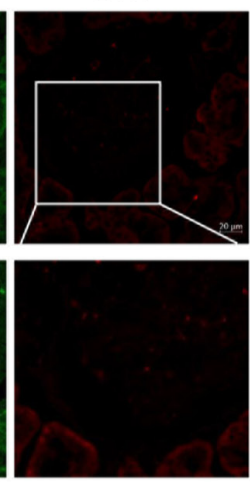

Control
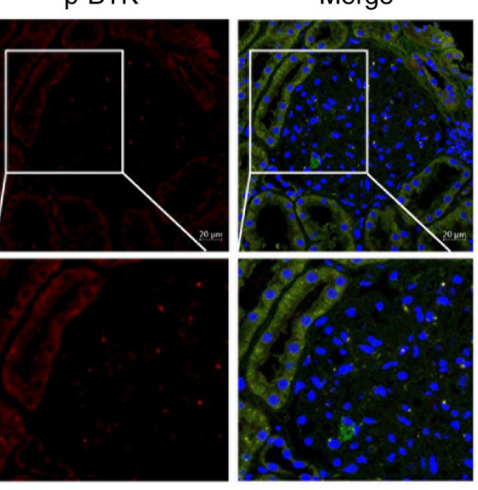

Merge
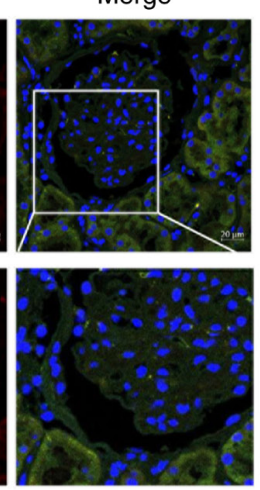
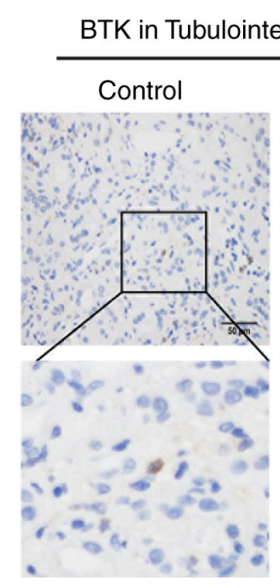

DN

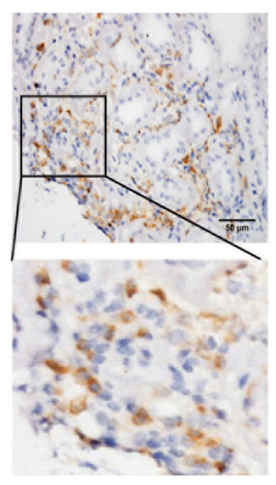

Glomerulus
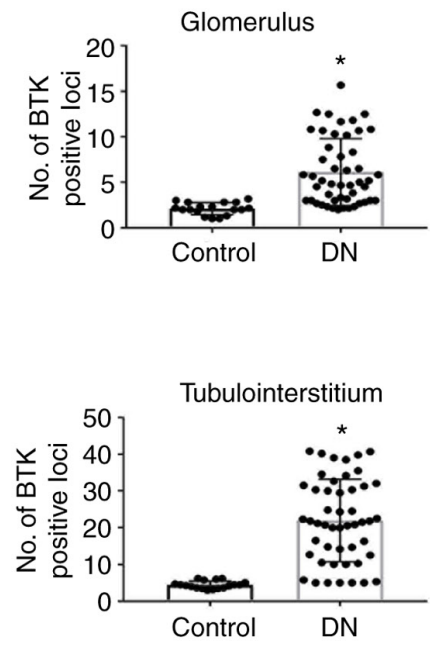

DN

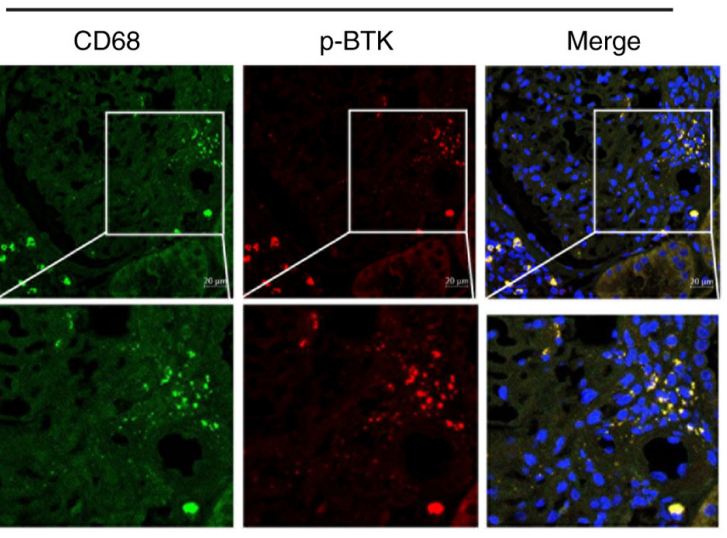

CD68

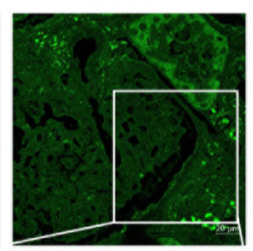

BTK
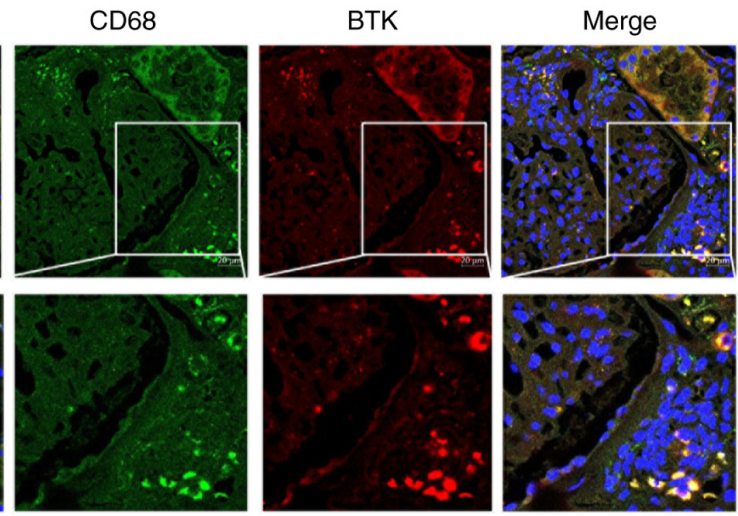

Figure 1. BTK is notably activated in renal macrophages in the kidneys of patients with DN. (A) Immunohistochemistry of BTK expression in the glomerulus and tubulointerstitium (scale bar, $50 \mu \mathrm{m} ;{ }^{*} \mathrm{P}<0.05$ vs. control group). (B) CD68 was co-stained with phosphorylated-BTK and BTK in the kidney (scale bar, $20 \mu \mathrm{m})$. BTK, Bruton's tyrosine kinase; DN, diabetic nephropathy.

WT1 and Nephrin were used to evaluate podocyte injury in the mouse kidney. It was found that the ColIV and FN expression levels were increased in the STZ group (Fig. 3A). Moreover, the numbers of WT1-positive cells and Nephrin-positive cells were significantly reduced (Fig. 3B). However, in the $\mathrm{BTK}^{-/}+\mathrm{STZ}$ group, the number of macrophages and the mesangial matrix were both decreased, and the number of podocytes was significantly increased. A reduced F4/80 expression was also observed in the $\mathrm{BTK}^{-/}+\mathrm{STZ}$ group, as compared with the STZ group (Fig. 3B). These results suggested that BTK knockout decreased macrophage infiltration and alleviated the accumulation of extracellular matrix and podocyte injury in diabetic mice.

Finally, TEM was used to observe the ultrastructural changes in the mouse kidneys. It was identified that, when compared with the WT group, the STZ group exhibited further extracellular matrix proliferation, thickened basement membrane and severe podocyte fusion. The number of podocytes was also decreased, and organelle loss was increased. However, these phenomena were all alleviated in the $\mathrm{BTK}^{-/}+$STZ group (Fig. 3C). Thus, these results indicated that BTK knockout alleviated renal injury in diabetic mice. 
A

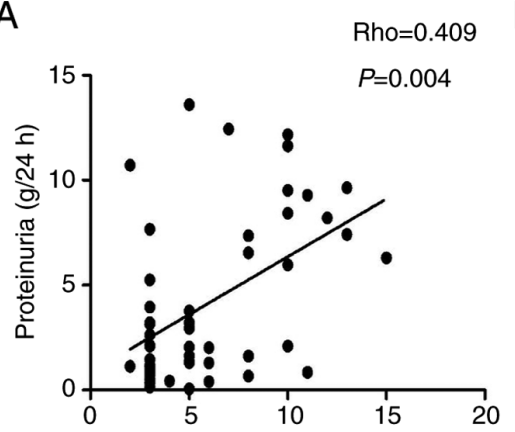

No. of BTK-positive loci in glomerulus
B

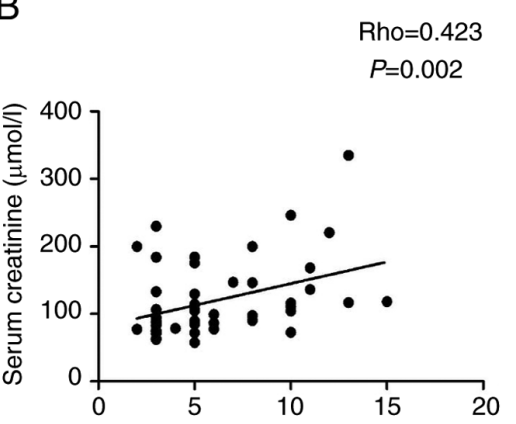

No. of BTK-positive loci in glomerulus
C

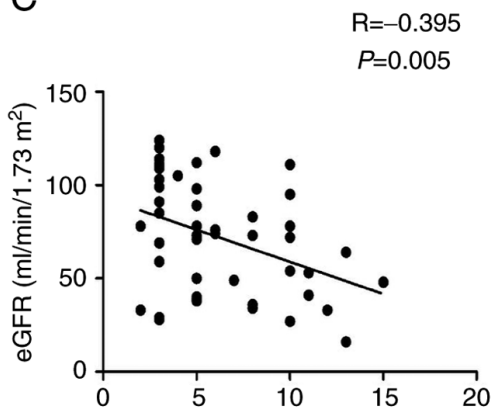

No. of BTK-positive loci in glomerulus
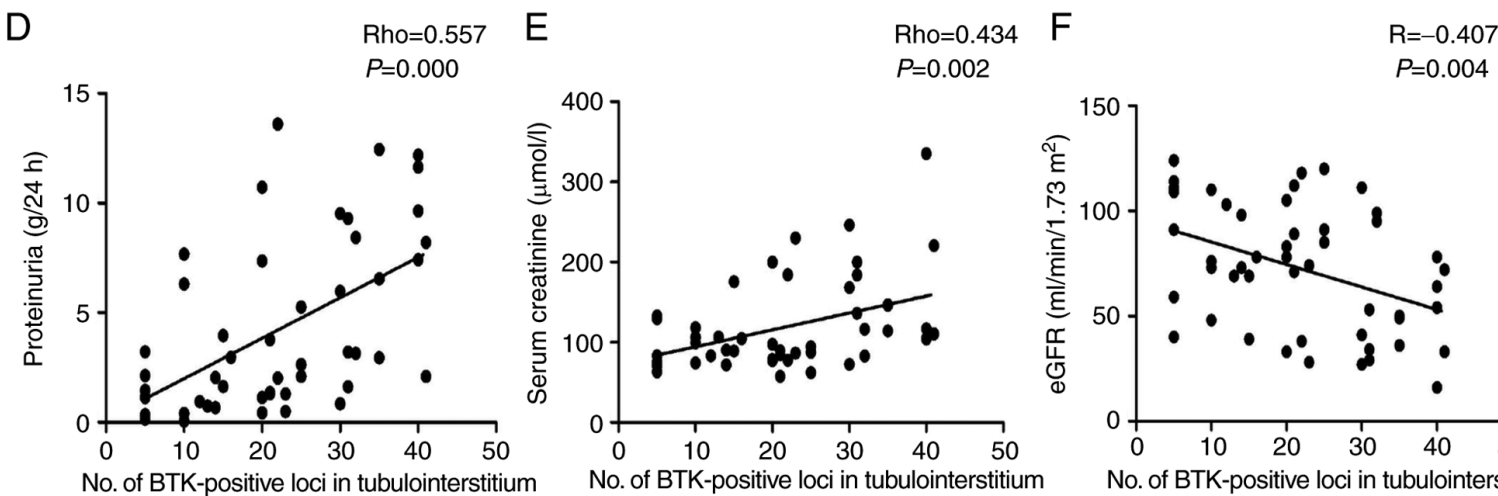

No. of BTK-positive loci in tubulointerstitium
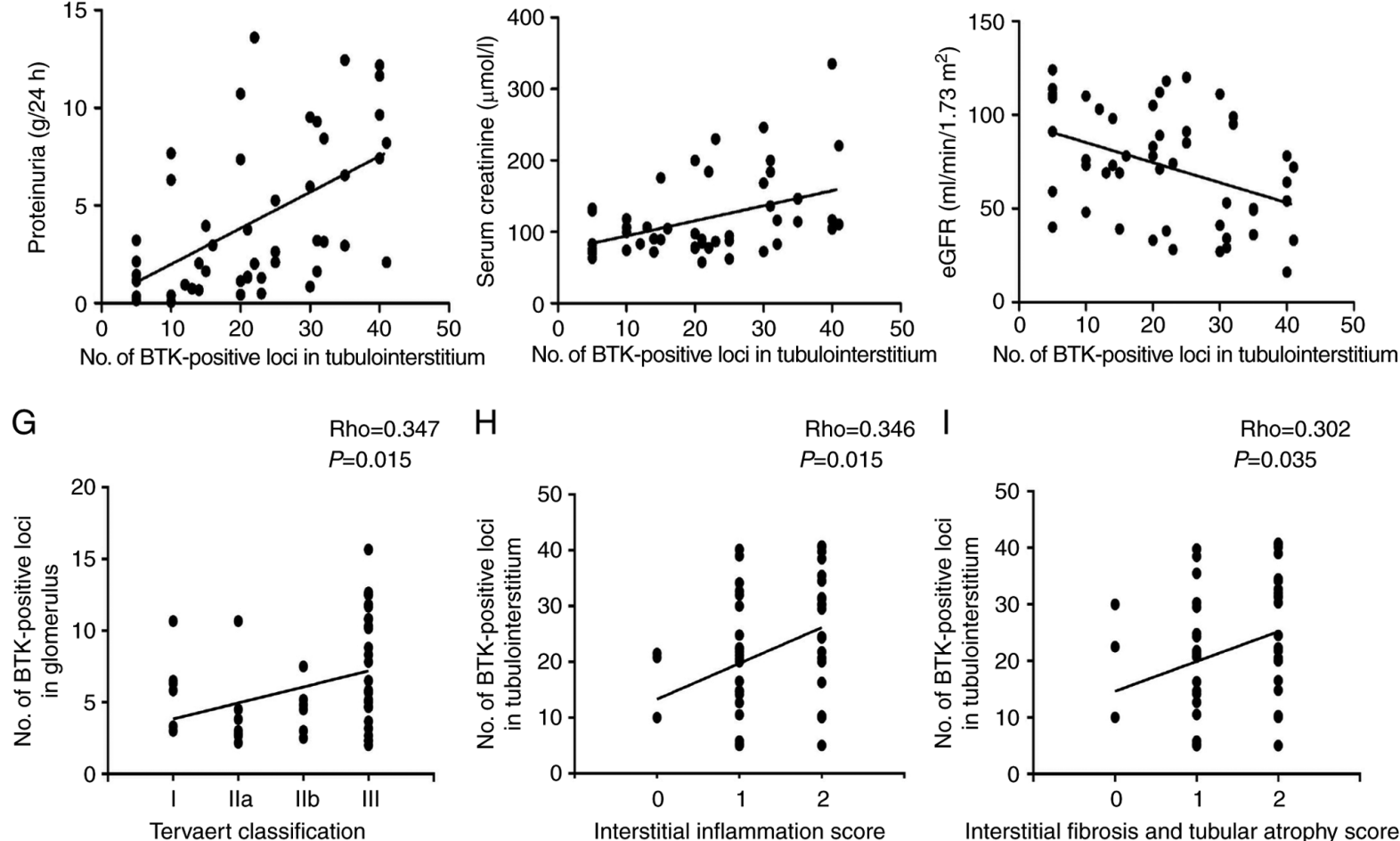

$\mathrm{H}$

Rho=0.346 I

$P=0.015$

Rho $=0.302$

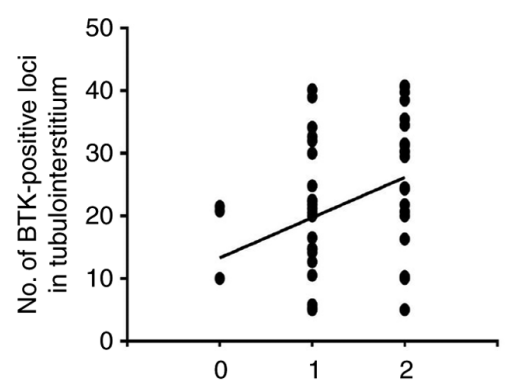

Interstitial inflammation score

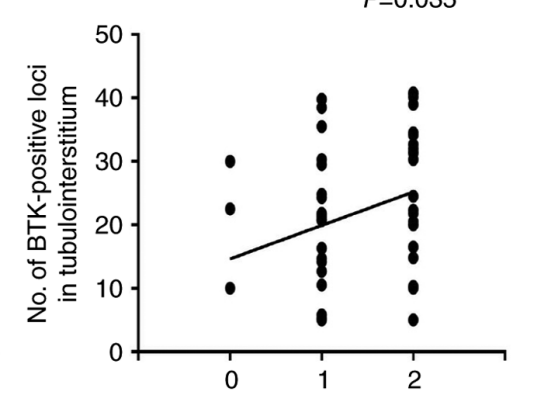

Interstitial fibrosis and tubular atrophy score

Figure 2. BTK expression is correlated with clinical indicators and pathological changes in the kidneys of patients with DN. (A) Correlation analysis of renal BTK expression in glomerulus with proteinuria in patients with type $2 \mathrm{DN}$. (B) Correlation analysis of renal BTK expression in glomerulus with serum creatinine in patients with type $2 \mathrm{DN}$. (C) Correlation analysis of renal BTK expression in glomerulus with eGFR in patients with type 2 DN. (D) Correlation analysis of renal BTK expression in tubulointerstitium with proteinuria, in patients with type 2 DN. (E) Correlation analysis between renal BTK expression in tubulointerstitium and serum creatinine in patients with type 2 DN. (F) Correlation analysis of renal BTK expression in tubulointerstitium with eGFR in patients with type 2 DN. (G) Correlation analysis between renal BTK expression in glomerulus and Tervaert classification in patients with type 2 DN. (H) Correlation analysis between renal BTK expression in tubulointerstitium and IFTA in patients with type 2 DN. (I) Correlation analysis of renal BTK expression between tubulointerstitium and interstitial inflammation in patients with type 2 DN. Pearson's test was used in (C and F) for correlation analysis. Spearman's test was used in (A, B, D, E, G H and I) for correlation analysis. BTK, Bruton's tyrosine kinase; DN, diabetic nephropathy; eGFR, estimated glomerular filtration rate; IFTA, interstitial fibrosis and tubular atrophy.

BTK knockout alleviates renal inflammation in diabetic mice. The change in the inflammatory response was also evaluated in the mice. The results of ELISA demonstrated that the TNF- $\alpha$, IL-1 $\beta$ and MCP-1 levels were increased in serum from the STZ group. However, this change was significantly reduced in the $\mathrm{BTK}^{-1-}+$ STZ group (Fig. 4A). Subsequently, changes in inflammatory mediators were detected in the mouse kidneys. The data indicated that the mRNA and protein expression levels of TNF- $\alpha$, IL- $1 \beta$ and MCP-1 were increased in the STZ group (Fig. 4B-D). Moreover, these changes were significantly reduced when BTK was knocked out. The immunohistochemistry results also revealed that the expression levels of TNF- $\alpha$, IL-1 $\beta$ and MCP-1 were significantly decreased in the $\mathrm{BTK}^{-/}+\mathrm{STZ}$ group compared with the STZ group (Fig. 4C). On the whole, the results indicated that diabetic mice exhibited additional severe inflammation and macrophage infiltration in serum and kidney, while BTK knockout alleviated this phenomenon.

BTK knockout suppresses the activation of the NLRP3 inflammasome in the kidneys of diabetic mice. To further observe the mechanisms of BTK in renal inflammation, WB analysis was conducted to observe the activation of the NLRP3 inflammasome in the mouse kidneys. The WB analysis data 


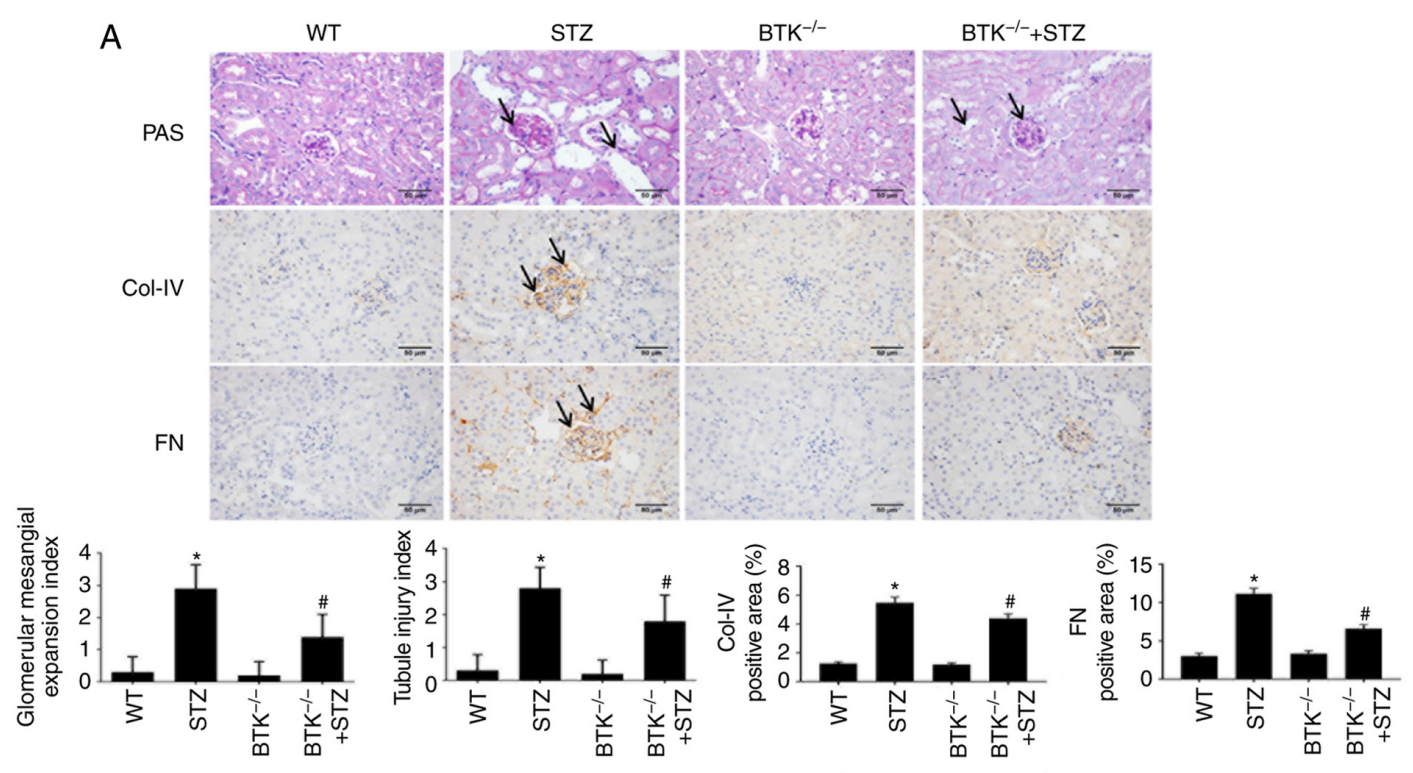

B

WT

STZ

$\mathrm{BTK}^{-1-}$

$\mathrm{BTK}^{-1-}+\mathrm{STZ}$
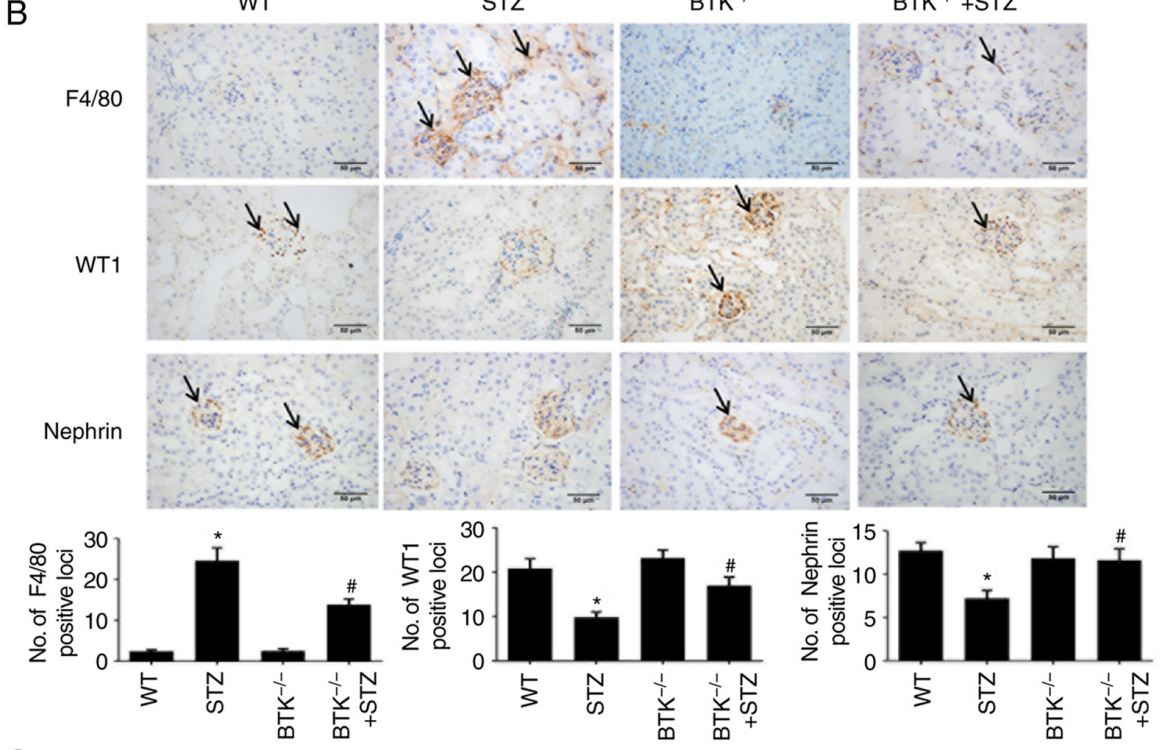

C

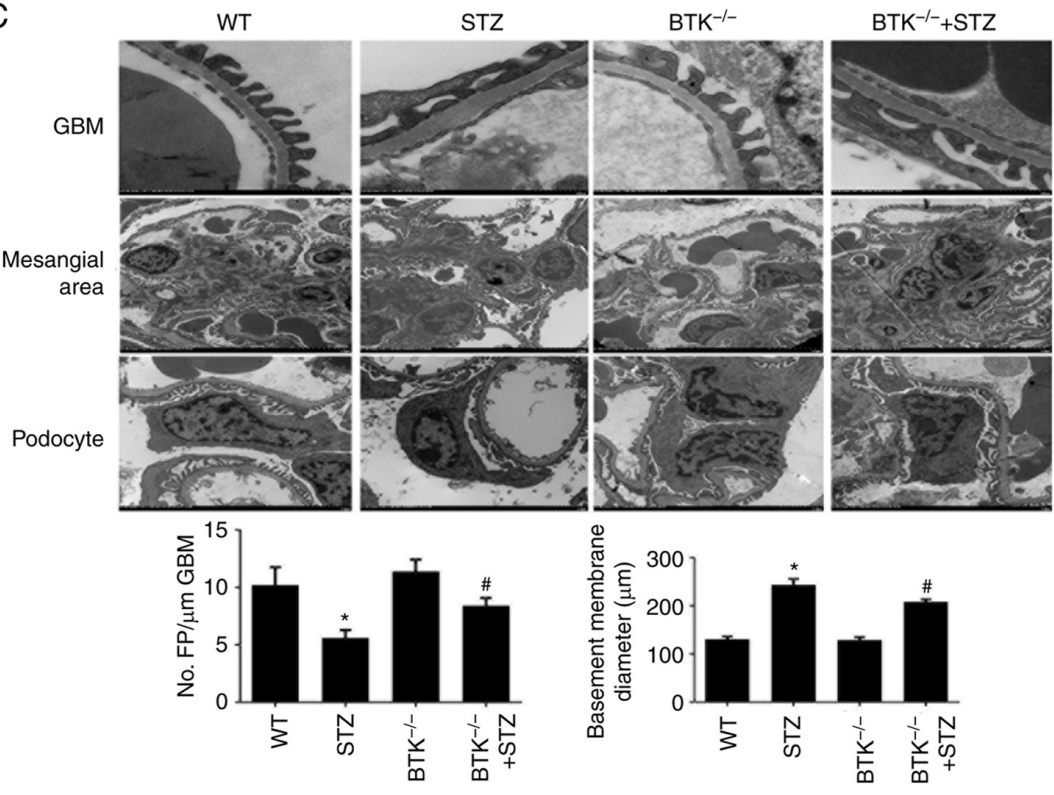

Figure 3. BTK knockout alleviates the pathological changes in mice with STZ-induced diabetes. (A) Glomerular mesangial expansion and tubular injury were detected using PAS staining. Immunohistochemistry of ColIV and FN expression was performed in mouse kidneys (scale bar, $50 \mu \mathrm{m}$ ). (B) Immunohistochemistry of F4/80, WT1 and FN expression levels in mouse kidneys (scale bar, $50 \mu \mathrm{m}$ ). (C) The thickness of GBM, mesangial expansion and podocyte injury were detected via transmission electron microscopy ("P<0.05 vs. WT group; ${ }^{*} \mathrm{P}<0.05$ vs. STZ group). WT, wild-type; BTK, Bruton's tyrosine kinase; FN, fibronectin; STZ, Streptozotocin; WT1, WT1 transcription factor; ColIV, collagen IV; PAS, periodic acid-Schiff; GBM, glomerular basement membrane. 
A
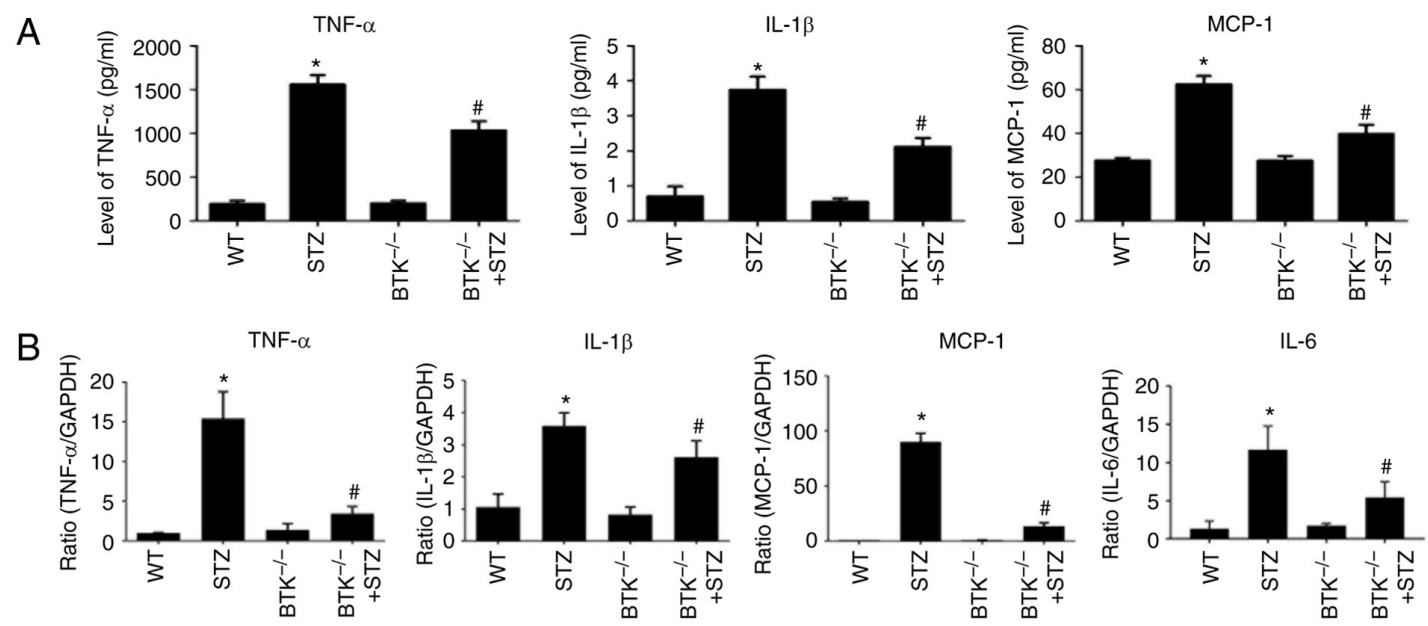

C

WT

STZ

$\mathrm{BTK}^{-1-}$

$\mathrm{BTK}^{-1-}+\mathrm{STZ}$
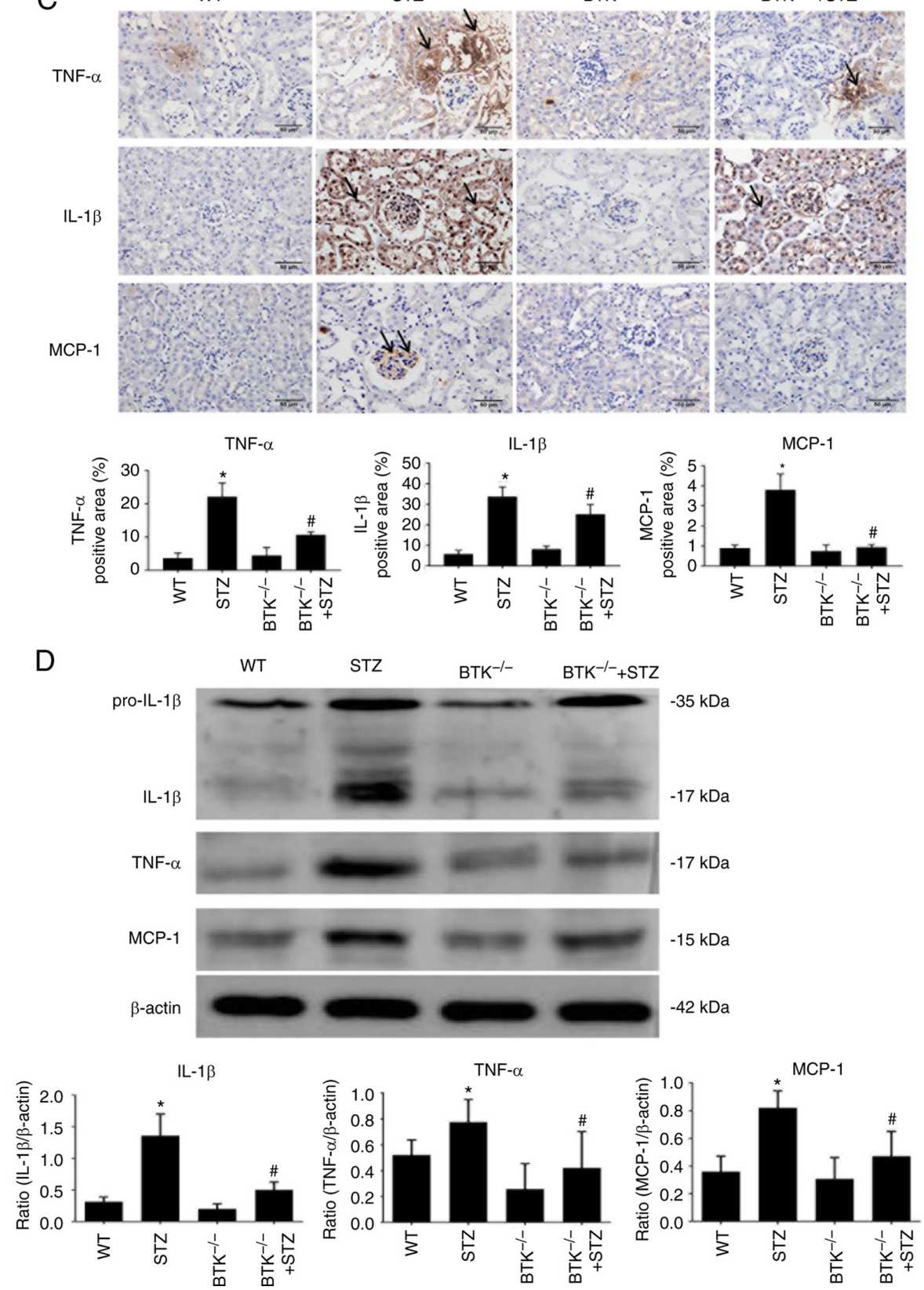

Figure 4. BTK knockout alleviates kidney inflammation in diabetic mice. (A) TNF- $\alpha$, IL-1 $\beta$ and MCP-1 levels of in mouse serum were detected using ELISA kits. (B) TNF- $\alpha$, IL-1 $\beta$, MCP-1 and IL-6 mRNA expression levels in mouse kidneys were detected by RT-qPCR. (C) TNF- $\alpha$, IL-1 $\beta$ and MCP-1 immunohistochemistry analysis of protein expression was conducted in mouse kidneys (scale bar, $50 \mu \mathrm{m}$ ). (D) Protein expression levels of TNF- $\alpha$, IL- $1 \beta$ and MCP- 1 were detected by western blot analysis ( $\mathrm{P}<0.05$ vs. WT group; ${ }^{\mathrm{P}} \mathrm{P}<0.05$ vs. STZ group). WT, wild-type; BTK, Bruton's tyrosine kinase; STZ, Streptozotocin; MCP-1, monocyte chemoattractant protein-1; RT-qPCR, reverse transcription-quantitative PCR. 
A

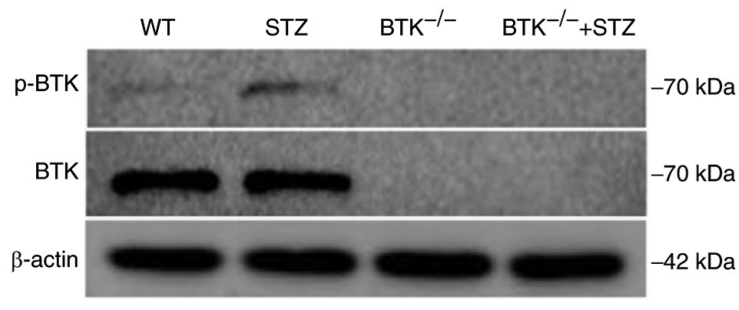

B

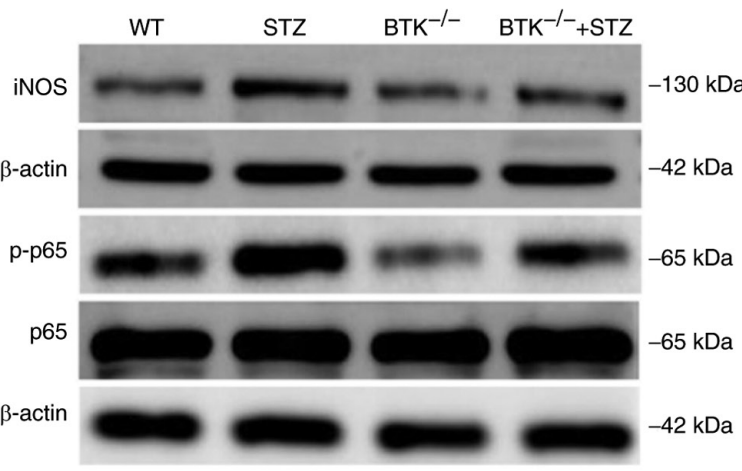

C
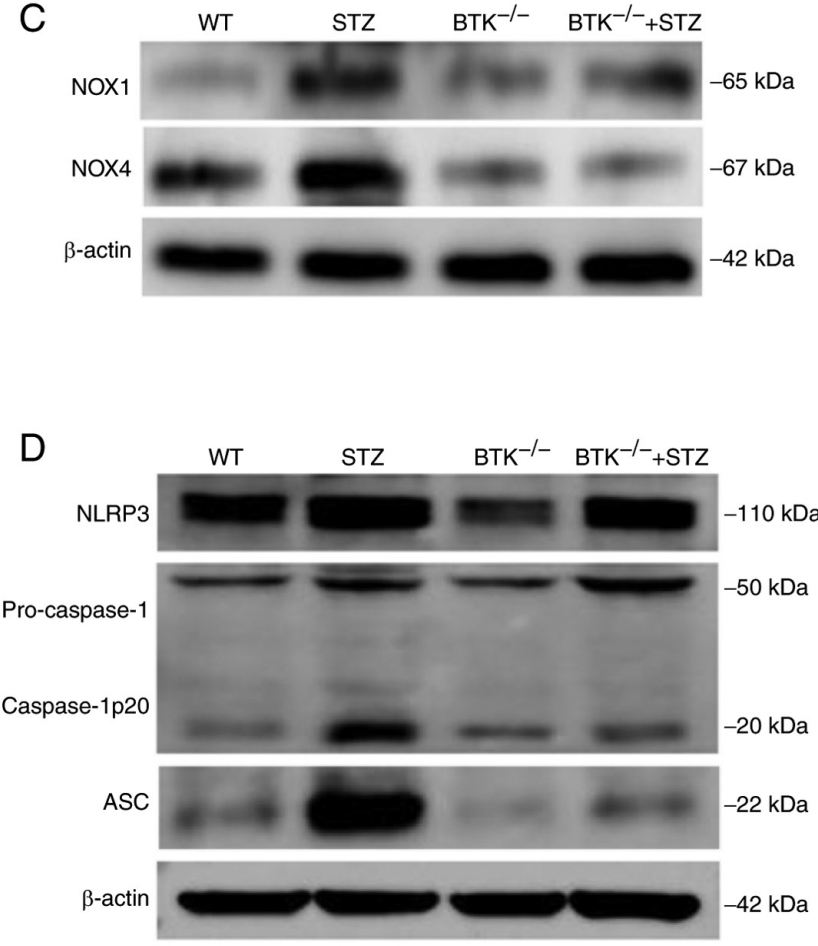
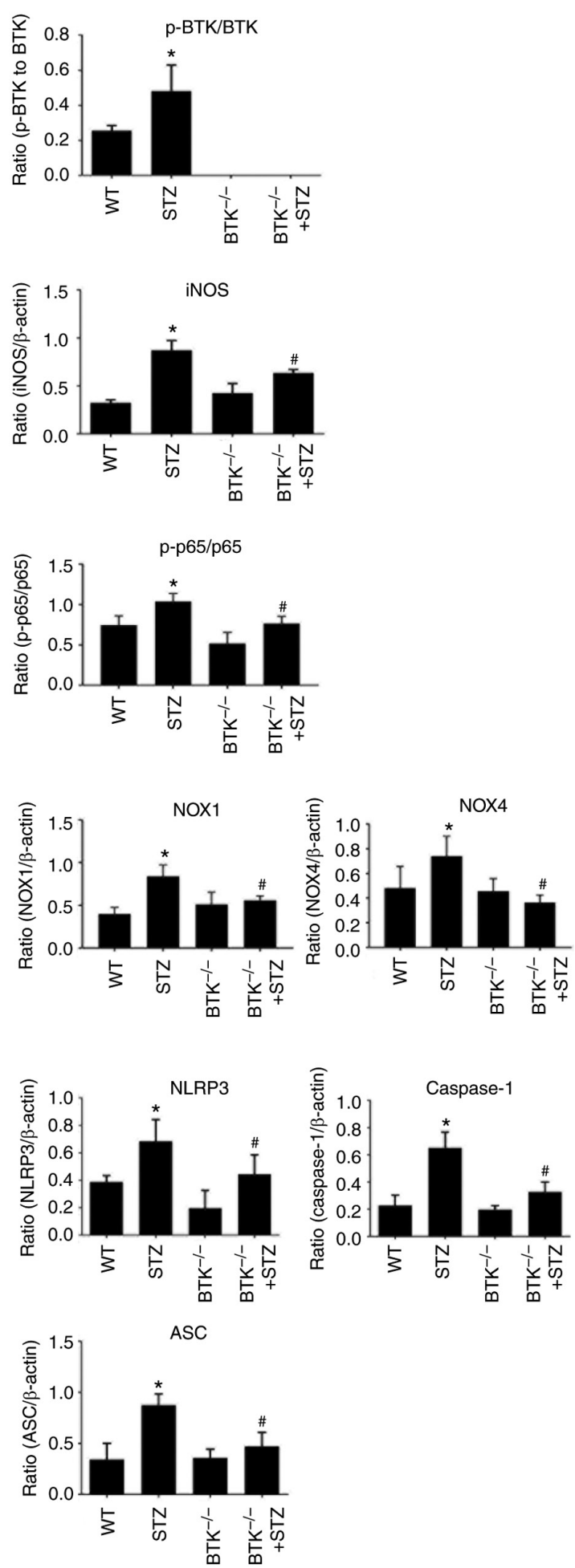

Figure 5. BTK knockout suppresses the oxidative stress and the activation of the NLRP3 inflammasome in the kidneys of diabetic mice. (A) p-BTK/BTK expression levels in mouse kidneys were detected via WB analysis. (B) iNOS, p-p65 and p65 expression levels in mouse kidneys were detected via WB analysis. (C) NOX1 and NOX4 expression levels in mouse kidneys were detected via WB analysis. (D) NLRP3, caspase-1 and ASC expression levels in mouse kidneys were detected via WB analysis ("P $<0.05$ vs. WT group; ${ }^{*} \mathrm{P}<0.05$ vs. STZ group). WT, wild-type; BTK, Bruton's tyrosine kinase; STZ, Streptozotocin; $\mathrm{p}-$-, phosphorylated; NLRP3, NLR family pyrin domain containing 3; iNOS, inducible nitric oxide synthase; ASC, apoptosis-associated speck-like protein containing a caspase recruitment domain; WB, western blot; p-p65, phosphorylated-NF-кB p65; NOX1, nicotinamide adenine dinucleotide phosphate oxidase 1; NOX4, nicotinamide adenine dinucleotide phosphate oxidase 4 .

demonstrated that the expression levels of p-BTK/BTK, iNOS and p-p65 were all increased in the STZ group. However, when BTK was knocked out, the expression levels of iNOS and p-p65 were significantly decreased (Fig. 5A and B). This suggested that BTK knockout reduced macrophage activation in the kidneys of diabetic mice. The change in oxidative stress in the mouse kidneys was also evaluated. According to these results, NOX1 and NOX4 expression levels were increased in 
the STZ group compared with the WT group. However, BTK deletion significantly alleviated this effect (Fig. 5C). Moreover, it was found that the expression levels of NLRP3, caspase-1 and ASC were significantly decreased in the $\mathrm{BTK}^{-/}+\mathrm{STZ}$ group compared with the STZ group (Fig. 5D). These results thus suggested that BTK knockout decreased oxidative stress and the activation of the NLRP3 inflammasome in the kidneys of diabetic mice.

\section{Discussion}

DN is a severe complication of diabetes mellitus, and its treatment remains challenging (1). Previous studies have reported that the development of DN is closely associated with inflammation, and that macrophages are the main immune cell type involved in this process $(5,6)$. In the early stages of DN, patients present an increase in serum inflammatory mediator levels. Subsequently, macrophages can be recruited into the kidneys by these mediators, such as $\mathrm{C}-\mathrm{C}$ motif chemokine ligand 2 and MCP-1 (34,35). This process was verified in the present study and culminated in an increased macrophage infiltration in kidneys. The results of the present demonstrated that either in the serum of patients with DN or in the serum of diabetic mice, inflammatory mediator expression levels were significantly increased. Moreover, macrophage infiltration and activation were increased in the kidneys of patients with $\mathrm{DN}$ and diabetic mice. It was also identified that the levels of inflammatory cytokines released by macrophages in kidneys were increased. These findings indicated that macrophages can be recruited into the kidneys and activated by hyperglycemia, leading to the aggravation of inflammation.

BTK is an important factor in innate immunity and has been closely associated with inflammation $(8,11)$. It has been previously revealed that high glucose levels may promote the phosphorylation of BTK, leading to the release of inflammatory mediators via the NF- $\kappa \mathrm{B}$ signaling pathway (15). It was also demonstrated in the present study that BTK was activated in renal macrophages in patients with DN. Moreover, BTK was associated with clinical indicators and pathological changes, which indicated that BTK played a crucial role in the disease progression of DN. In in vivo experiments, it was identified that BTK knockout reduced UAER, the accumulation of extracellular matrix and macrophage infiltration in diabetic mice. The fibrotic lesions were also observed by Masson's staining, although no significant changes were observed in any of the groups analyzed. This may be attributed to an early diabetic pathology change in mouse kidneys observed at 12 weeks of feeding, which manifested through mesangial matrix proliferation and tubulointerstitial damage. Podocytes are important intrinsic cells in the kidney and are known as the most vulnerable cells in the kidney (36). Podocyte loss and fusion of foot process can occur in early stage DN, and it is also the main cause of proteinuria $(37,38)$. Therefore, it was considered that podocyte injury could be a favorable choice to evaluate early renal injury, thus WT1 and Nephrin were used the present study as podocyte markers (39). The results of the present study demonstrated that BTK knockout protected the diabetic mice from podocyte injury, further confirmed through TEM. It was also noted that the thickened basement membrane, proliferated extracellular matrix and podocyte injury were alleviated in BTK knockout diabetic mice. Collectively, these results suggested that BTK knockout alleviated kidney injury in diabetic mice.

The NLRP3 inflammasome is a multiprotein complex that regulates innate immune responses to infection and cell stress (18). In recent studies, it was reported that the activation of NLRP3 may be associated with disease progression in patients with DKD $(21,22)$, Currently, there are no existing clinically approved therapies that target the NLRP3 inflammasome directly (24). BTK has been proven to serve as a critical regulator of NLRP3 inflammasome activation (25). It has been observed that the regulatory effects of BTK inhibition on the NLRP3 inflammasome vary in different mouse models. BTK deficiency has been shown to promote NLRP3 inflammasome activation and induce IL-1 $\beta$-mediated colitis, but it also alleviates other diseases, such as ischemic brain injury, diet-induced metabolic inflammation and polymicrobial sepsis (26-28). On this basis, the association between BTK and the NLRP3 inflammasome in DN was examined in the present study. It was revealed that no significant side-effects were noted in BTK knockout mice. Moreover, it was noted that BTK knockout markedly reduced the NLRP3, caspase-1 and ASC expression levels in the kidneys of diabetic mice. Those results suggested that the protective effect of BTK knockout was mainly attributed to the reduced activation of the NLRP3 inflammasome.

In conclusion, the present study demonstrated that there was a notable association between BTK and the clinical indicators and pathological changes of patients with DN. Moreover, BTK knockout reduced inflammation in diabetic mice, and this protection was a result of the suppression of NLRP3 inflammasome activation. However, the lack of analysis of IL-6 protein and $\mathrm{NF}-\kappa \mathrm{B}$ mRNA expression also present a limitation of the present study. To date, to the best of our knowledge, there is no effective treatment available for DN. BTK inhibitor had been widely used in clinical practice. The present study demonstrated that BTK deletion effectively reduced the activation of the NLRP3 inflammasome; thus, this may prove to be a potential method for the treatment of DN. The findings presented herein may have important clinical significance; BTK inhibitor may be an effective therapeutic strategy against DN. The authors aim to perform further studies in the future in order to provide further suggestions which may aid in the development of novel treatment strategies for DN.

\section{Acknowledgements}

The authors would like to thank the Research and Experimental Center of Anhui Medical University, for providing experimental technical support.

\section{Funding}

The present study was supported financially by the National Natural Science Foundation of China (grant no. 81470965) and the Natural Science Foundation of Anhui Province (grant no. $1808085 \mathrm{MH} 236)$.

\section{Availability of data and materials}

All data generated or analyzed during this study are included in this published article. 


\section{Authors' contributions}

JZ and YGW conceived and designed the study. JC and YYL collected the data and performed the animal experiments. JZ and JC analyzed the data and wrote the manuscript. YGW and LLX conducted the animal experiments and reviewed the manuscript. JZ, LLX and YGW confirmed the authenticity of all the raw data. All authors have read and approved the final manuscript.

\section{Ethics approval and consent to participate}

All patients and healthy individuals had signed informed consent forms and all experiments were approved by the Ethics Committee of Anhui Medical University (approval number: 5101309). The animal experiments were performed in accordance with guidelines, 'Principles of Laboratory Animal Care and Use in Research' (Ministry of Health, Beijing, China). The experiments were approved by the Anhui Medical University Ethics Committee (approval number: 2020064).

\section{Patient consent for publication}

Not applicable.

\section{Competing interests}

The authors declare that they have no competing interests.

\section{References}

1. Cole JB and Florez JC: Genetics of diabetes mellitus and diabetes complications. Nat Rev Nephrol 16: 377-390, 2020.

2. Tervaert TW, Mooyaart AL, Amann K, Cohen AH, Cook HT, Drachenberg CB, Ferrario F, Fogo AB, Haas M, de Heer E, et al: Pathologic classification of diabetic nephropathy. J Am Soc Nephrol 21: 556-563, 2010.

3. Liang G, Song L, Chen Z, Qian Y, Xie J, Zhao L, Lin Q, Zhu G, Tan Y, Li X, et al: Fibroblast growth factor 1 ameliorates diabetic nephropathy by an anti-inflammatory mechanism. Kidney Int 93 : 95-109, 2018.

4. Guilliams M, Thierry GR, Bonnardel J and Bajenoff M: Establishment and maintenance of the macrophage niche. Immunity 52: 434-451, 2020.

5. Klessens CQF, Zandbergen M, Wolterbeek R, Bruijn JA, Rabelink TJ, Bajema IM and IJpelaar DHT: Macrophages in diabetic nephropathy in patients with type 2 diabetes. Nephrol Dial Transplant 32: 1322-1329, 2017.

6. Fu J, Akat KM, Sun Z, Zhang W, Schlondorff D, Liu Z, Tuschl T, Lee K and He JC: Single-Cell RNA profiling of glomerular cells shows dynamic changes in experimental diabetic kidney disease. J Am Soc Nephrol 30: 533-545, 2019.

7. Smith CI, Islam TC, Mattsson PT, Mohamed AJ, Nore BF and Vihinen M: The Tec family of cytoplasmic tyrosine kinases: Mammalian Btk, Bmx, Itk, Tec, Txk and homologs in other species. BioEssays 23: 436-446, 2001.

8. Weber ANR, Bittner Z, Liu X, Dang TM, Radsak MP and Brunner C: Bruton's Tyrosine Kinase: An emerging key player in innate immunity. Front Immunol 8: 1454, 2017.

9. Pal Singh S, Dammeijer F and Hendriks RW: Role of Bruton's tyrosine kinase in B cells and malignancies. Mol Cancer 17: 57, 2018.

10. Haselmayer P, Camps M, Liu-Bujalski L, Nguyen N, Morandi F, Head J, O'Mahony A, Zimmerli SC, Bruns L, Bender AT, et al: Efficacy and pharmacodynamic modeling of the BTK inhibitor evobrutinib in autoimmune disease models. J Immunol 202 : 2888-2906, 2019.

11. Rip J, de Bruijn MJW, Appelman MK, Pal Singh S, Hendriks RW and Corneth OBJ: Toll-Like receptor signaling drives BTK-mediated autoimmune disease. Front Immunol 10: 95, 2019.
12. Wei J, Wang Y, Qi X and Wu Y: Enhanced Bruton's tyrosine kinase activity in the kidney of patients with $\operatorname{IgA}$ nephropathy. Int Urol Nephrol 53: 1399-1415, 2021.

13. Kong W, Deng W, Sun Y, Huang S, Zhang Z, Shi B, Chen W, Tang X, Yao G, Feng X and Sun L: Increased expression of Bruton's tyrosine kinase in peripheral blood is associated with lupus nephritis. Clin Rheumatol 37: 43-49, 2018.

14. Jain N, Keating M, Thompson P, Ferrajoli A, Burger J, Borthakur G, Takahashi K, Estrov Z, Fowler N, Kadia T, et al: Ibrutinib and venetoclax for first-line treatment of CLL. N Engl J Med 380: 2095-2103, 2019.

15. Fan Z, Wang Y, Xu X and Wu Y: Inhibitor of Bruton's tyrosine kinases, PCI-32765, decreases pro-inflammatory mediators' production in high glucose-induced macrophages. Int Immunopharmacol 58: 145-153, 2018.

16. Roschewski M, Lionakis MS, Sharman JP, Roswarski J, Goy A, Monticelli MA, Roshon M, Wrzesinski SH, Desai JV, Zarakas MA, et al: Inhibition of Bruton tyrosine kinase in patients with severe COVID-19. Sci Immunol 5: eabd0110, 2020.

17. Rathinam VA and Fitzgerald KA: Inflammasome complexes: Emerging mechanisms and effector functions. Cell 165: 792-800, 2016.

18. Wang L and Hauenstein AV: The NLRP3 inflammasome: Mechanism of action, role in disease and therapies. Mol Aspects Med 76: 100889, 2020.

19. Hooftman A, Angiari S, Hester S, Corcoran SE, Runtsch MC, Ling C, Ruzek MC, Slivka PF, McGettrick AF, Banahan K, et al: The immunomodulatory metabolite itaconate modifies NLRP3 and inhibits inflammasome activation. Cell Metab 32: 468-478.e7, 2020.

20. Liu D, Yang P, Gao M, Yu T, Shi Y, Zhang M, Yao M, Liu Y and Zhang X: NLRP3 activation induced by neutrophil extracellular traps sustains inflammatory response in the diabetic wound. Clin Sci (Lond) 133: 565-582, 2019.

21. Han Y, Xu X, Tang C, Gao P, Chen X, Xiong X, Yang M, Yang S, Zhu X, Yuan S, et al: Reactive oxygen species promote tubular injury in diabetic nephropathy: The role of the mitochondrial ros-txnip-nlrp3 biological axis. Redox Biol 16: 32-46, 2018.

22. Mulay SR: Multifactorial functions of the inflammasome component NLRP3 in pathogenesis of chronic kidney diseases. Kidney Int 96: 58-66, 2019.

23. Tang SCW and Yiu WH: Innate immunity in diabetic kidney disease. Nat Rev Nephrol 16: 206-222, 2020.

24. Ram C, Jha AK, Ghosh A, Gairola S, Syed AM, Murty US, Naidu VGM and Sahu BD: Targeting NLRP3 inflammasome as a promising approach for treatment of diabetic nephropathy: Preclinical evidences with therapeutic approaches. Eur J Pharmacol 885: 173503, 2020.

25. Weber ANR: Targeting the NLRP3 inflammasome via BTK. Front Cell Dev Biol 9: 630479, 2021

26. Ito M, Shichita T, Okada M, Komine R, Noguchi Y, Yoshimura A and Morita R: Bruton's tyrosine kinase is essential for NLRP3 inflammasome activation and contributes to ischaemic brain injury. Nat Commun 6: 7360, 2015.

27. Purvis GSD, Collino M, Aranda-Tavio H, Chiazza F, O'Riordan CE, Zeboudj L, Mohammad S, Collotta D, Verta R, Guisot NE, et al: Inhibition of Bruton's TK regulates macrophage $\mathrm{NF}-\kappa \mathrm{B}$ and NLRP3 inflammasome activation in metabolic inflammation. Br J Pharmacol 177: 4416-4432, 2020.

28. O'Riordan CE, Purvis GSD, Collotta D, Krieg N, Wissuwa B, Sheikh MH, Ferreira Alves G, Mohammad S, Callender LA, Coldewey SM, et al: X-Linked immunodeficient mice with no functional bruton's tyrosine kinase are protected from sepsis-induced multiple organ failure. Front Immunol 11: 581758, 2020.

29. Li XQ, Chang DY, Chen M and Zhao MH: Deficiency of C3a receptor attenuates the development of diabetic nephropathy. BMJ Open Diabetes Res Care 7: e000817, 2019.

30. Liu J, Lee GY, Biggers JD, Toth TL and Toner M: Low cryoprotectant concentration rapid vitrification of mouse oocytes and embryos. Cryobiology 98: 233-238, 2021.

31. Weinerman R, Ord T, Bartolomei MS, Coutifaris C and Mainigi M: The superovulated environment, independent of embryo vitrification, results in low birthweight in a mouse model. Biol Reprod 97: 133-142, 2017.

32. Livak KJ and Schmittgen TD: Analysis of relative gene expression data using real-time quantitative PCR and the 2(-Delta Delta C(T)) method. Methods 25: 402-408, 2001.

33. Birnbaum Y, Bajaj M, Yang HC and Ye Y: Combined SGLT2 and DPP4 Inhibition Reduces the Activation of the Nlrp3/ASC inflammasome and attenuates the development of diabetic nephropathy in mice with type 2 diabetes. Cardiovasc Drugs Ther 32: 135-145, 2018. 
34. Moreno JA, Gomez-Guerrero C, Mas S, Sanz AB, Lorenzo O, Ruiz-Ortega M, Opazo L, Mezzano S and Egido J: Targeting inflammation in diabetic nephropathy: A tale of hope. Expert Opin Investig Drugs 27: 917-930, 2018.

35. Wen Y and Crowley SD: The varying roles of macrophages in kidney injury and repair. Curr Opin Nephrol Hypertens 29: 286-292, 2020.

36. Nagata M: Podocyte injury and its consequences. Kidney Int 89: 1221-1230, 2016

37. Agrawal S, He JC and Tharaux PL: Nuclear receptors in podocyte biology and glomerular disease. Nat Rev Nephrol 17: 185-204, 2021.
38. Zhou L, Chen X, Lu M, Wu Q, Yuan Q, Hu C, Miao J, Zhang Y, $\mathrm{Li} \mathrm{H}$, Hou FF, et al: Wnt/ $\beta$-catenin links oxidative stress to podocyte injury and proteinuria. Kidney Int 95: 830-845, 2019.

39. Puelles VG, Bertram JF and Moeller MJ: Quantifying podocyte depletion: Theoretical and practical considerations. Cell Tissue Res 369: 229-236, 2017.

c) (i) (9) This work is licensed under a Creative Commons

EY No ND Attribution-NonCommercial-NoDerivatives 4.0 International (CC BY-NC-ND 4.0) License. 